Review Article

\title{
Cardiac Manifestations in COVID-19 Patients: A Focus on the Pediatric Population
}

\author{
Tania Abi Nassif $\mathbb{D},{ }^{1}$ Ghina Fakhri $\mathbb{D},{ }^{1}$ Nour K. Younis $\mathbb{D},{ }^{2}$ Rana Zareef $\mathbb{D},{ }^{1}$ \\ Farah Al Amin (D), Fadi Bitar $\mathbb{D}^{3},{ }^{3}$ and Mariam Arabi $\mathbb{D}^{3}$ \\ ${ }^{1}$ Department of Pediatrics and Adolescent Medicine, American University of Beirut Medical Center, Beirut, Lebanon \\ ${ }^{2}$ Faculty of Medicine, American University of Beirut Medical Center, Beirut, Lebanon \\ ${ }^{3}$ Division of Cardiology, Department of Pediatrics and Adolescent Medicine, American University of Beirut Medical Center, \\ Beirut, Lebanon
}

Correspondence should be addressed to Mariam Arabi; ma81@aub.edu.lb

Received 16 February 2021; Accepted 28 June 2021; Published 17 July 2021

Academic Editor: Louis Detolla

Copyright (c) 2021 Tania Abi Nassif et al. This is an open access article distributed under the Creative Commons Attribution License, which permits unrestricted use, distribution, and reproduction in any medium, provided the original work is properly cited.

Background. SARS-CoV-2 is a new strain of the coronavirus family that emerged by the end of 2019 and led to the unpreceded COVID-19 pandemic. The virus affects multiple organs simultaneously and leads to a high rate of morbidity and mortality in all age groups. The cardiovascular system is one of the major affected organ systems. Various mechanisms including direct myocardial injury contribute to the cardiac manifestations of COVID-19 patients. Methods. We performed a comprehensive and updated search on the cardiac manifestations of COVID-19. Our search included laboratory and imaging evaluations. In addition, we added a unique section on the effect of SARS-CoV-2 on the cardiovascular system in the pediatric population. Results. COVID19 might have an effect on the cardiovascular system at various levels leading to myocardial ischemia, arrhythmia, heart failure, myocarditis, and multisystem inflammatory syndrome in children. The incidence of cardiovascular complications varies among patients. This paper also provides a comprehensive summary of all the reported pediatric cases with cardiac manifestations. Conclusion. Multidisciplinary teams are crucial for adequate management of patients with COVID-19 regardless of age. Timely diagnosis is critical in reducing mortality.

\section{Introduction}

Coronaviruses have previously recorded two large epidemics in 2003 and 2012, respectively [1, 2]. In December 2019, an outbreak of atypical pneumonia was witnessed in Wuhan, China. The causative agent of this outbreak was a novel coronavirus, named severe acute respiratory syndrome coronavirus 2 (SARS-CoV-2). Later, the World Health Organization (WHO) acknowledged the disease related to this virus as coronavirus disease-19 (COVID-19). In March of 2020, COVID-19 was declared to be a world pandemic [3].

SARS-CoV-2 is an enveloped positive-sense singlestranded RNA virus. It spawns a wide spectrum of diseases affecting multiple organs. By early May 2020, the virus had already reached over 200 countries worldwide afflicting at least 4 million victims. As of February 10, 2021, there have been more than 106 million confirmed cases of COVID-19 and more than 2 million deaths around the globe $[3,4]$.

Despite being of zoonotic origin, SARS-CoV-2 exhibits human-to-human transmission through the droplet, contact, and ocular routes. It can remain viable for 3 hours as an aerosol and for 72 hours on certain surfaces. The virus mainly invades the upper respiratory tract leading to respiratory symptoms. However, multisystem involvement is observed as well, including the cardiovascular system. Indeed, the natural history of COVID-19 proved that the cardiovascular system may act as a primary target for SARSCoV-2 and thus may be influenced directly by the virus. Similarly, secondary sequelae of the disease provoke an indirect injury to the cardiovascular system. Therefore, it is 
reasonably valid that patients with underlying cardiovascular morbidities are at increased risk of experiencing severe disease and a higher mortality rate $[5,6]$.

The virus can directly invade cells of various origins including the pulmonary, cardiac, and intestinal cells. Subsequently, tissues are injured through direct viral invasion and indirect release of cytokines and inflammatory markers. The exaggerated inflammatory response accompanied by the cytokine storm is a key player in the multiorgan involvement of the disease. Initially, most patients present with fever, dry cough, and shortness of breath. As the disease progresses, patients may develop nausea, vomiting, abdominal pain, and diarrhea [6]. Around 10\% of patients experience severe disease and require intensive care and supplemental oxygen therapy. These patients are at increased risk of developing acute respiratory distress syndrome with diffuse alveolar damage, cardiac injury, viremia, superimposed bacterial infections, and multiorgan failure [7].

Owed to the rapid development of events, a considerable amount of data has become available in the literature to explain the pathophysiology and clinical features of COVID19. However, only a few reports provide a comprehensive review of the cardiovascular manifestations. Besides, when it comes to COVID-19 in the pediatrics population, the literature is scarce and limited. This is largely because children exhibit milder disease forms. In this paper, we provide a comprehensive review of the cardiovascular manifestations of COVID-19, with a special focus on the pediatric population.

\section{Renin-Angiotensin-Aldosterone System}

The renin-angiotensin-aldosterone system (RAAS) is a complex system involved in regulating blood pressure and maintaining fluid and electrolyte balance. It can aggravate or precipitate atherosclerosis leading to myocardial hypertrophy and fibrosis [8]. Renin cleaves angiotensin to angiotensin I (AT1) which is cleaved by angiotensin-converting enzyme (ACE) to angiotensin II (AT2). Two receptors for AT2 with similar affinities exert a wide array of different cascading effects. ACE-2, a monologue of ACE, cleaves AT2 back to angiotensin 1-7. Angiotensin 1-7 exerts anti-inflammatory, antiproliferative, and vasodilating effects. The family of coronaviruses has a well-established mechanism of binding to ACE2 receptor, thus mediating its proteolytic modification and entry into the cell [9]. The outer spike S-proteins are found abundantly on the surface of the virus bind with high affinity to ACE2 receptor [10]. The infection is initiated when the virus is internalized into the cells via clathrin-coated pits. Its viral RNA is subsequently released into the cytoplasm. Zhou et al. reported that SARS-CoV-2 can only enter ACE2-expressing host cells to initiate the infection process [11]. These findings suggest that a shared receptor, ACE-2, plays a role in both determining the fate of AT2 and mediating the cytotoxicity of SARS-CoV-2. Hence, AT2 may be potentially influenced by SARS-CoV-2 internalization and intracellular replication, and in a similar manner, SARS-CoV-2 pathogenicity may be modulated by AT2 fate.

\section{COVID-19 Pathophysiology}

The pathophysiology of COVID-19 is reflected by three phases. The first phase is triggered by viral penetration into the respiratory epithelium and is followed by cellular proliferation. The initial immune response is marked by the activation of monocytes and macrophages and is portrayed by mild symptoms. The next phase begins with pulmonary vasodilatation and increased vascular permeability. Leukocyte migration then ensues leading to fluid extravasation and pulmonary edema. Consequently, alveolar damage, hypoxemia, cardiac damage, and stress are precipitated. The final phase is characterized by a massive cytokine storm secondary to the exaggerated inflammatory response $[12,13]$.

3.1. A Hyperinflammatory State. The excessive release of proinflammatory cytokines engenders a dramatically amplified immune response against the virus. Indeed, the plasma levels of interleukin-2 (IL2), interleukin-6 (IL-6), tumor necrosis factor $\alpha(\mathrm{TNF} \alpha)$, and C-reactive protein (CRP) are markedly elevated in COVID-19 patients. They are considered key contributors in the process leading to multiorgan failure [14]. Besides, the virus can downregulate the expression of ACE2 leading to a cascade of events and multiorgan manifestations summarized in Figure 1. The innate immune system plays a pivotal role in the uncontrolled inflammatory response mounted against the virus. The internalization and the reduction of expression of ACE2 receptor on cellular surface result in enhanced levels of circulating Ang II. Ultimately, a vicious cycle, maintained by ever-increasing inflammatory markers, is developed $[10,15]$.

ACE2 is extensively expressed on the entire circulatory system, especially the coronary vessels. Vascular smooth muscles cells and endothelial cells present in both the arterial and venous systems express ACE2 on their surfaces. Viral entry and replication are potent triggers of an exaggerated immune response characterized by a cytokine storm and eventually by endothelial activation and dysfunction. The inflamed endothelium soon becomes dysfunctional and predisposes to a proinflammatory prothrombotic state [16].

\section{SARS-CoV-2 and the Pediatric Population}

The Chinese Center for Disease Control reported that $<1 \%$ of the patients with COVID-19 were under the age of 10 years [12]. In another large pediatric-only cohort in China, authors reported that children tend to have a less severe form of COVID-19 with the majority presenting with mild symptoms. However, infants with COVID-19 were in a critical condition compared to older children [17]. The reason behind milder disease presentation in children is not yet clear. Some theories support the notion that children have a less functional ACE2 receptor compared to adults; thus, the infectivity of the virus is decreased. Another theory argues that children have higher levels of ACE2 which in its turn reduces Ang II plasma levels. Ang II is recognized to correlate strongly with the clinical course of COVID-19 


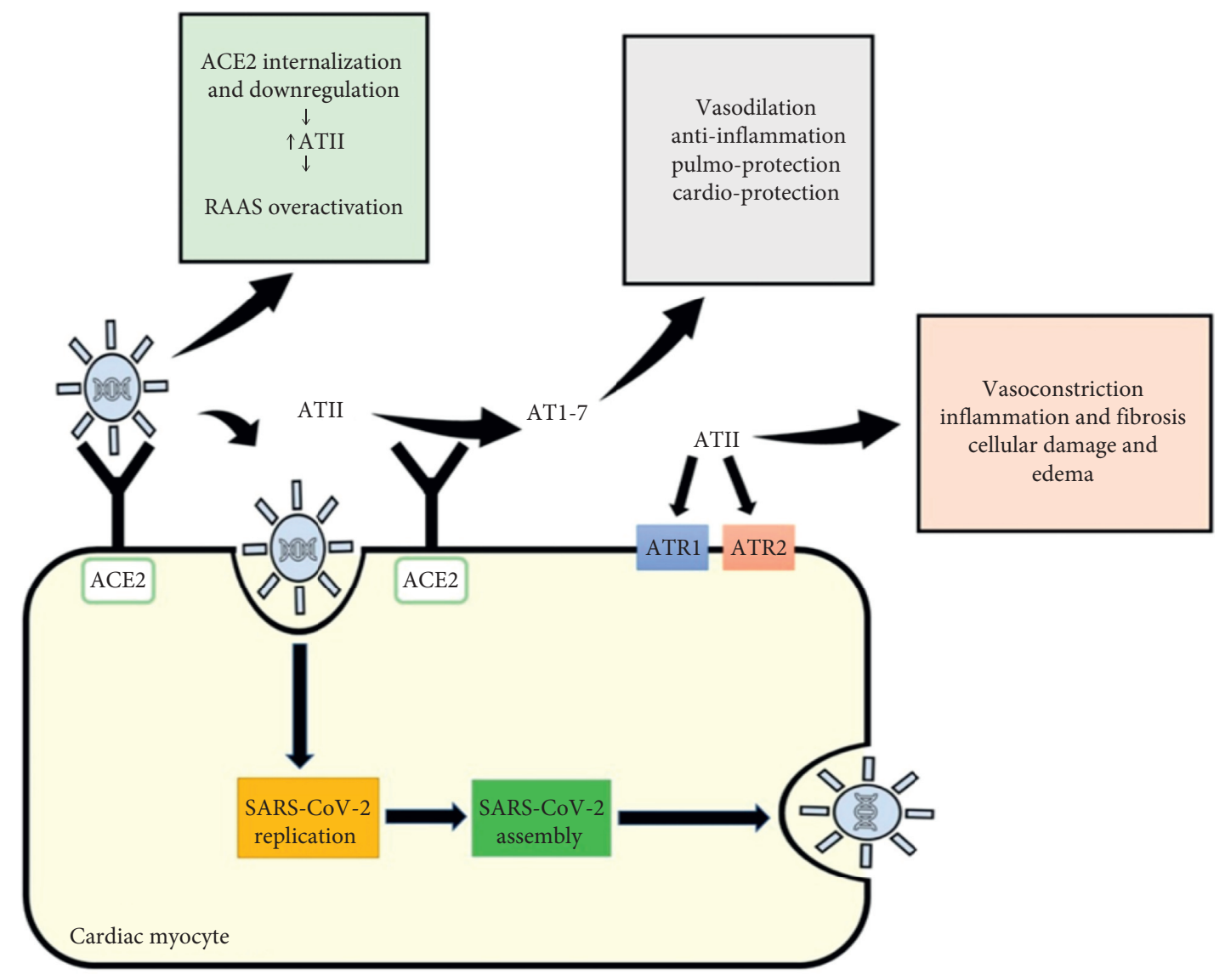

FIgURE 1: An Interplay between angiotensin II and SARS-CoV-2. SARS-CoV-2 cellular uptake is mediated primarily by the ACE2 receptor. Viral internalization is then followed by viral replication, assembly, and release. ACE2 is involved in converting angiotensin II (ATII) into angiotensin 1-7 (AT1-7). Unlike ATII, AT1-7 has anti-inflammatory, cardioprotective, and pulmoprotective effects with vasodilatory properties. Interestingly, SARS-CoV-2 binding to ACE2 provokes ACE2 internalization and downregulation. Consequently, this increases the circulatory levels of ATII and upregulates numerous proinflammatory, fibrotic, and vasoconstrictory pathways.

disease [6]. Indeed, children infected with COVID-19 have low hospital admission rates and an estimated mortality rate of less than $5 \%$ [18]. However, a minority of children tend to exhibit serious disease manifestations, especially cardiovascular injury. Table 1 depicts a comprehensive summary of the reported cardiac-related signs and symptoms of COVID19 disease in children.

\section{SARS-CoV-2 and the Heart}

There are three mechanisms that lead to cardiac involvement in the setting of COVID-19: (1) direct injury caused by direct viral entry to myocardial cells, (2) hypoxia-induced myocardial ischemia, and (3) heightened exaggerated inflammatory response characterized by endothelial overactivation and microvascular thrombi $[32,33]$. The virus exerts a direct effect on the myocardial cells by directly binding to the ACE2 receptors present on these cells leading to direct myocardial injury [34]. The beneficial role of ACE2 and angiotensin on the heart is well documented. Their interaction inhibits oxidative stress and cardiac remodeling and at the same time induces coronary vasorelaxation $[35,36]$. It has been demonstrated that ACE2 expression increases at the initial phases of myocardial injury. In fact, ACE2 knocked-out animal models experienced exaggerated myocardial hypertrophy and heart failure. In COVID-19, there is a substantial downregulation of ACE2, and the subsequently elevated levels of angiotensin result in the overactivation of RAAS. Hence, the protective effect of angiotensin is lost, and myocardial injury is precipitated. Interestingly, elderly individuals are found to have lower levels of ACE2 expression, which could partially explain the poor outcome in this age group [37]. Moreover, there is a unanimous agreement that patients with underlying cardiovascular comorbidities are at a higher risk of contracting SARS-CoV-2 and developing severe disease. These patients also have significantly increased mortality rates [21, 38-41]. This is attributed to the reduced baseline cardiovascular reserve and to the ischemic injury prompted by the viral infection.

In a similar manner, the inflammatory markers correlate with electrocardiographic abnormalities and cardiac injury [42]. The elevation of inflammatory markers is accompanied by a surge in cardiac biomarkers and is associated with cardiac injury. Sequentially, worse clinical presentation and increased morbidity and mortality are encountered [43]. In a 


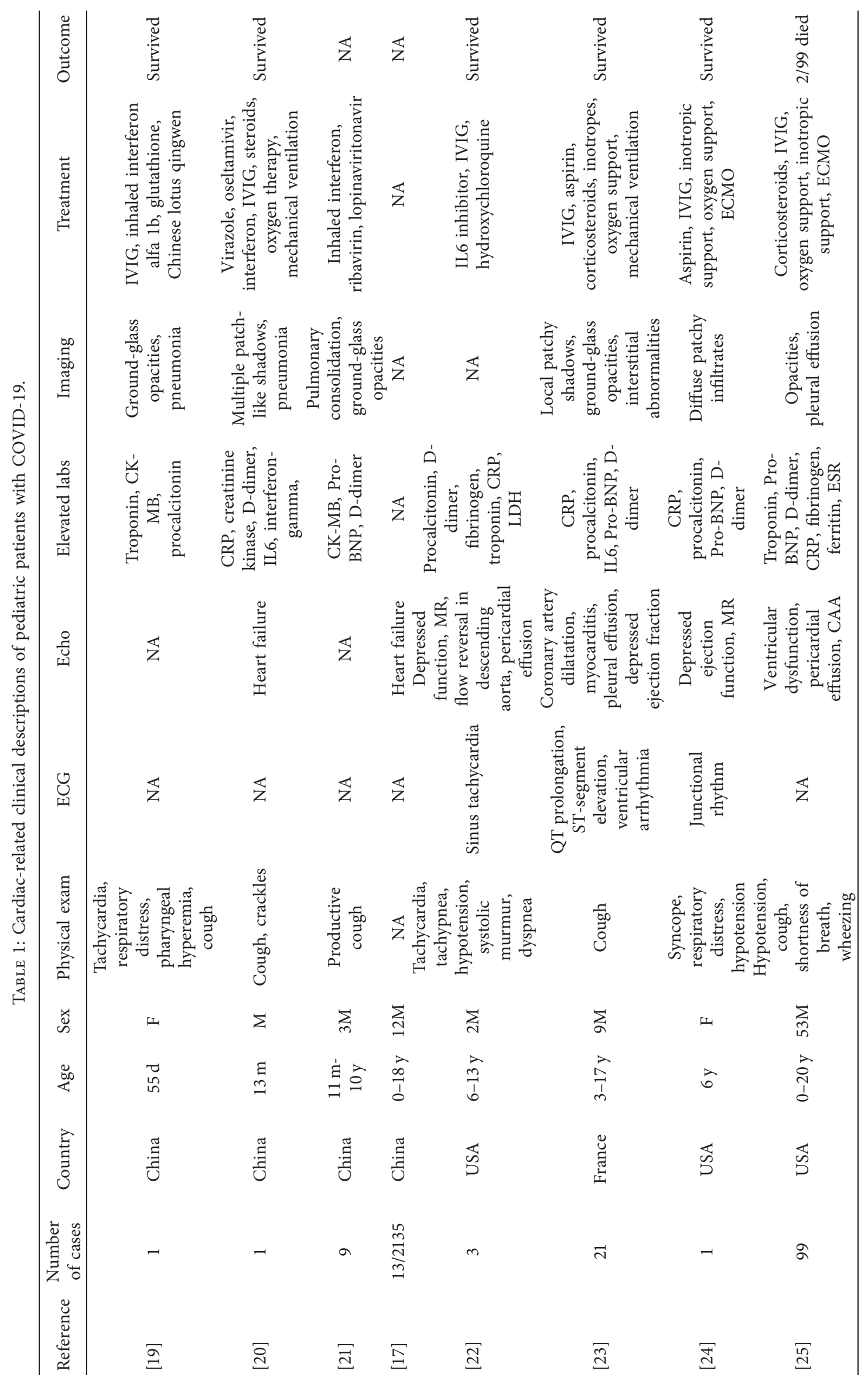




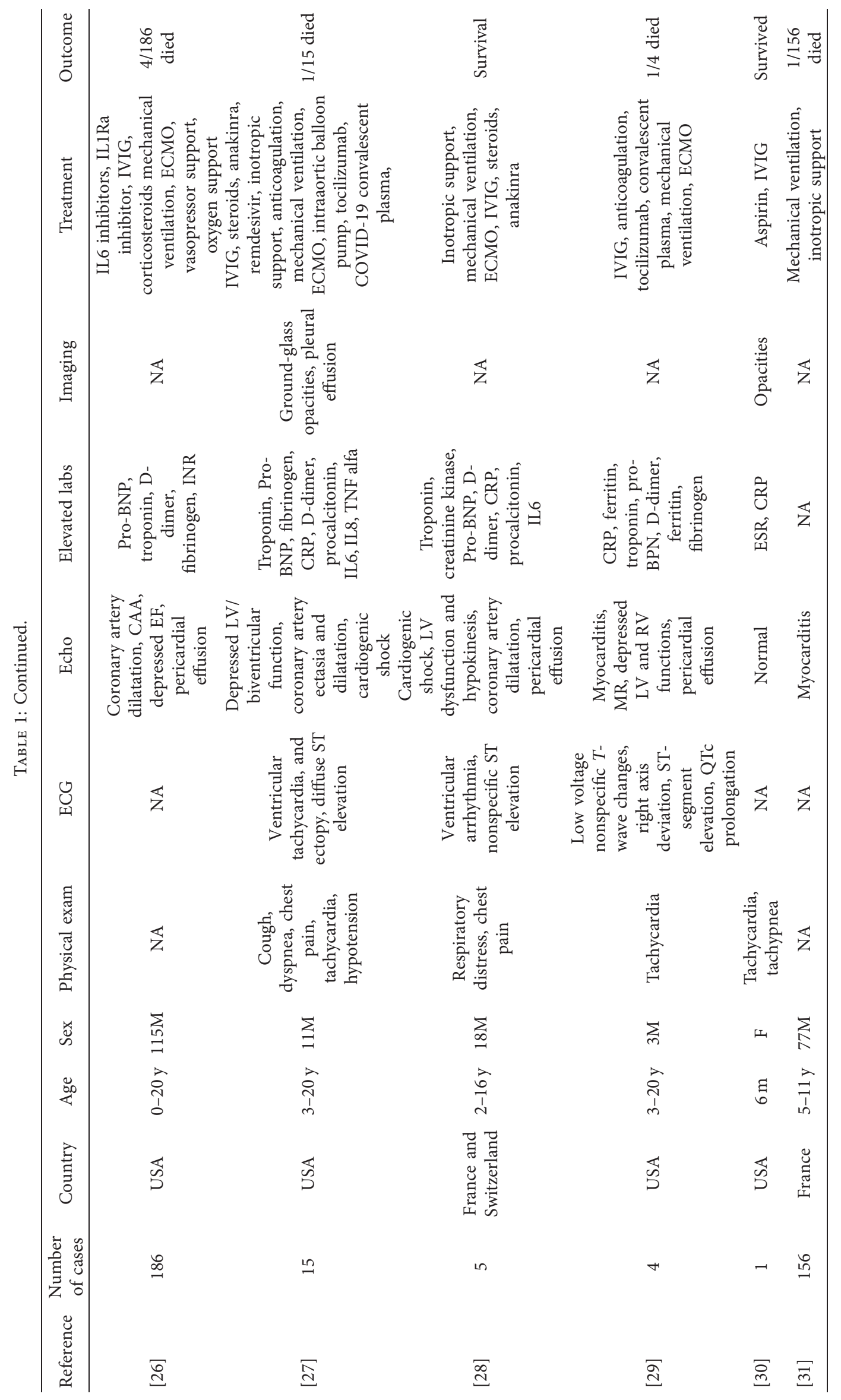


retrospective cohort in China earlier this year, the authors reported that the elevation of cardiac biomarkers was associated with increased mortality from COVID-19 [44]. Multiple studies have also demonstrated that patients with underlying cardiovascular diseases such as hypertension and coronary artery disease are at an increased risk to contract severe forms of COVID-19 and are at substantial risk of death. In a recent meta-analysis, Li et al. revealed that the relative risk of contracting severe COVID-19 and requiring intensive care was 2.03 (95\% CI 1.54-2.68, $p<0.00001)$ and 3.3 (95\% CI 2.03-5.36, $p<0.00001)$ in patients with hypertension and coronary artery disease, respectively [45].

Oudit et al. performed autopsies on patients with confirmed SARS infection. He reported that $35 \%$ of these patients had the virus's genome embedded in the DNA of the myocardial cells. The same subset of patients had a more aggressive course during their illness as compared to patients without cardiac involvement [46]. Edema of the cardiac stroma, atrophy of myocardial fibers, and inflammatory cells infiltration were also observed. Early acute involvement of the myocardial system was unanimously associated with higher morbidity and mortality [5-7, 45, 47]. Considering the strong homology between different types of SARS viruses, we can safely assume that SARS-CoV-2 may also invade the myocardial cells in a similar manner. Several studies have confirmed the presence of the SARS-CoV-2 genome integrated into the human myocardium [48-50]. In a recent report of 104 patients with COVID-19, endomyocardial biopsy confirmed that 5 of them had the SARSCoV-2 genome in the myocardial tissues. Histologic examination revealed the presence of myocardial necrosis, inflammation, and microvascular thrombi [51]. In a study with a larger cohort, the authors documented the presence of the SARS-CoV-2 genome in almost $65 \%$ of the patients with COVID-19 [52]. Unanimously, it was found that a higher myocardial viral load is linked to a higher expression of proinflammatory cytokines [49-52].

5.1. Type I MI: Acute Myocardial Injury. Type I myocardial injury is defined by the acute elevation in cardiac troponin levels with or without evidence of myocardial ischemia on an electrocardiogram. The presence of acute myocardial injury among COVID-19 patients was identified as a significant factor associated with a 3.4-increased risk of death. Similarly, it is estimated that one-third to one-fifth of hospitalized patients with COVID-19 will have evidence of acute myocardial injury. Additionally, the presence of myocardial injury is also associated with a higher need for mechanical ventilation [53-55].

Myocardial infarction, under the umbrella of acute myocardial injury, is caused by the dislodgement of an atherosclerotic plaque in individuals with atherosclerosis. Viral products can predispose to plaque displacement by binding to and activating immune receptors on cells present within the plaque. This cascade of infection and inflammation causes coronary endothelial dysfunction and thrombosis $[56,57]$.
5.2. Type II Myocardial Injury. Type II MI emanates from an imbalance in myocardial oxygen demand and supply. In the setting of COVID-19, type II MI may arise from several contributory factors: (1) the presence of an atherosclerotic plaque that predisposes to gradual reduction in blood flow, (2) a dysfunctional endothelium, (3) elevated levels of ATII, (4) hypertension and vasoconstriction, and (5) hypoxemia [58-60]. During states of severe stress and increased inflammation, SARS-CoV-2 infection can lead to myocardial ischemia and infarction by disrupting the balance between myocardial oxygen supply and demand. This imbalance may be present with or without the effect of an atherosclerotic plaque, owing to the great physiological stress triggered by the infection.

The incidence of myocardial ischemia in the general COVID-19 population ranges between $7 \%$ and $40 \%$, which reflects the heterogeneity of the studied population $[14,42,43,61]$.

Heart failure and myocardial damage contributed to approximately $40 \%$ of the deaths of SARS-CoV-2-positive patients. This mortality risk is significantly higher than the one associated with older age and prior medical diseases [44].

In adults, injury to the myocardium and subsequent troponin elevation was reported in up to $12 \%$ of SARS-CoV2 -infected patients $[6,62]$. In another case series, the incidence of myocardial injury reached $7.2 \%$ [42]. In the pediatric population, myocardial injury in patients under 20 years of age was reported in terms of elevation of CK-MB, troponin, and Pro-BPN [19-21, 38-41, 63, 64]. In another large prospective study done exclusively on pediatric patients, at least $73 \%$ of the enrolled patients had elevated cardiac biomarkers. 50\% of them had elevated troponin alone while the majority had increased Pro-BNP levels above $400 \mathrm{pg} / \mathrm{mL}$ [26].

In addition to the increased mortality risk, elevated troponin levels also correlate with a worse clinical picture (higher need for mechanical ventilation), as well as higher levels of other biomarkers such as IL-6, CRP, and D-dimer $[32,43]$. Patients with myocardial injury and elevated troponin levels were consistently older than patients without myocardial injury. This phenomenon has not yet been reported in the pediatric population. The most common echocardiographic finding in patients with any element of myocardial injury is right ventricular dilatation and dysfunction. Left ventricular dysfunction is less commonly reported in the literature. Focal and diffuse ST-segment elevations are also common findings on electrocardiograms [65-67].

5.3. Heart Failure. Patients commonly present with tachycardia, which later leads to impaired diastolic filling and subsequent systolic and diastolic dysfunction. Eventually, mortality increases when patients present with hypotension and heart failure. A large cohort study reported that up to 7\% of patients with SARS-CoV-2 had an element of left ventricular diastolic dysfunction with ensuing low cardiac output. On the other hand, in this same cohort, left 
ventricular systolic dysfunction was reported in more $>50 \%$ of patients [63].

In one of the largest cohort studies in China limited to the pediatric population, 13 out of the 2135 patients who tested positive for SARS-CoV-2 had cardiac involvement [17]. Depressed ejection fraction is reported in most studies that assessed the cardiac function of the involved children. An ejection fraction as low as $10 \%$ was reported in one of the prospective studies in France [23]. In a large prospective study in pediatric patients with SARS-CoV-2, the authors reported that severe heart failure with ejection fraction $<30 \%$ was present in $5 \%$ of the patients. Moreover, while the majority had an ejection fraction above $55 \%$, ejection fraction declined to $30 \%-55 \%$ in a third of the children [26]. Another large prospective cohort study reported a degree of heart failure in the pediatric population. The mean of the left ventricular ejection fraction was found to be between 10 and $57 \%$, with a median of $38 \%$ [23]. This suggests that SARSCoV-2 may result in both diastolic and systolic myocardial failure not only in adults but also in pediatric patients.

5.4. Myocarditis. Myocarditis is defined as inflammation in the myocardium of the heart. Once present, it can cause dysfunction in the cardiac muscle, disruption in the electrical system, and reduction in cardiac contractility. The incidence is currently unknown in patients with COVID-19, as the evidence in the literature is limited to case reports [68-70]. In a cohort with small sample size, cardiac magnetic resonance imaging performed at a median of 71 days after the SARS-CoV-2 infection revealed myocarditis in $80 \%$ of the patients [71-73]. In the pediatric population, myocardial dysfunction has also been described. In a large prospective study in France, more than half of the children enrolled had evidence of myocarditis with depressed ejection fraction and elevated inflammatory and cardiac biomarkers. There was evidence of pericardial effusion as well [23]. Echocardiographic findings include a large left ventricular volume, left ventricular diastolic dysfunction, and low left ventricular ejection fraction. MRI findings often include a late gadolinium enhancement, a raised native $\mathrm{T} 1$ and $\mathrm{T} 2$, and pericardial enhancement [71].

In a large pediatric cohort study, myocarditis was noted in $71 \%$ of the admitted patients [23]. This suggests that SARS-CoV-2 induces myocarditis, myocardial ischemia, and heart failure in a significant proportion of the infected patients.

5.5. Arrhythmia. There are different types of arrhythmias described in the setting COVID-19 infection. The long list includes but not limited to sinus tachycardia, atrial arrhythmia, first-degree atrioventricular block, nonsustained ventricular tachycardia and fibrillation, premature atrial and ventricular beats, and incomplete right bundle branch blocks [21, 40, 41]. In the pediatric population, the following arrhythmias were reported: atrial arrhythmia, atrioventricular block, and bundle branch blocks [41]. The estimated incidence rate of arrhythmia in COVID-19 patients is around 17\% [62]. The Heart Rhythm Society has recently pointed out that in addition to the direct myocardial injury and ensuing arrhythmia, electrolyte disturbances are quite common and can induce arrhythmias themselves. Despite these hypotheses, the exact mechanism behind the initiation of any kind of arrhythmia in the setting of COVID-19 infection is still unclear. In another large cohort, atrioventricular block occurred in $28.7 \%$ of adult patients hospitalized for COVID-19. Sinus tachycardia was reported in $19.6 \%$. In general, arrhythmia was reported in more than $50 \%$ of the patients in one cohort [63]. On the other hand, in a larger case series, only $16.7 \%$ of the hospitalized patients developed arrhythmia [42]. In a more recent large prospective study, pediatric SARS-CoV-2 positive patients had an incidence rate of arrhythmias of $12 \%$ [26].

5.6. Cardiomyopathy. The severe systemic inflammation in COVID-19 can precipitate cardiotoxicity and lead to cardiovascular dysfunction. Patients with sepsis-induced cardiomyopathy tend to have elevated circulating levels of inflammatory markers such as IL- 6 and TNF- $\alpha$ [74, 75]. In vitro studies have shown that exposure to elevated levels of IL-6 and TNF- $\alpha$ led to left ventricular systolic dysfunction and reduced contractility [76]. In another study, the incidence of cardiomyopathy was reported to reach $33 \%$ in patients infected with the virus [6]. In a large cohort, up to $10 \%$ of patients hospitalized for COVID-19 were reported to have tricuspid, aortic, or mitral regurgitation leading to hemodynamic changes [63]. Moreover, cardiac dysfunction was reported in $41.2 \%$ of patients hospitalized for COVID19 in a large cohort study. While less commonly reported in the pediatric population, in several cohort studies, the authors reported the presence of left ventricular dysfunction in patients under the age of 20 years. Left ventricular dilatation, trace mitral regurgitation, and hypokinesia of the inferior left ventricular wall were particularly reported in these patients [38-40].

5.7. Multisystem Inflammatory Syndrome in Children (MIS-C). In April 2020, the National Health Services in the United Kingdom reported the first case of MIS-C. At the time, it was a new combination of atypical Kawasaki disease and toxic shock syndrome under the umbrella of severe COVID-19. Later, the WHO and the United States Center for Disease Control and Prevention set criteria for diagnosis with overlapping features [77]. The pathophysiology involves a sequela of events. The first one is denoted by acute necrotizing arteritis characterized by neutrophils infiltrating the vessel walls. This is followed by the formation of an aneurysm within the coronary artery. Macrophages and T cell lymphocytes infiltrate the damaged vessel wall to begin a chronic form of vasculitis. Over the years, myofibroblast proliferation leads to coronary artery stenosis. In the early acute phase, myocardial edema can develop leading to myocarditis before evidence of an aneurysm. A transient left ventricular dysfunction may occur and lead to cardiovascular shock in some patients [73].

Patients who develop MIS-C amidst their SARS-CoV-2 infection are usually older than patients who develop 


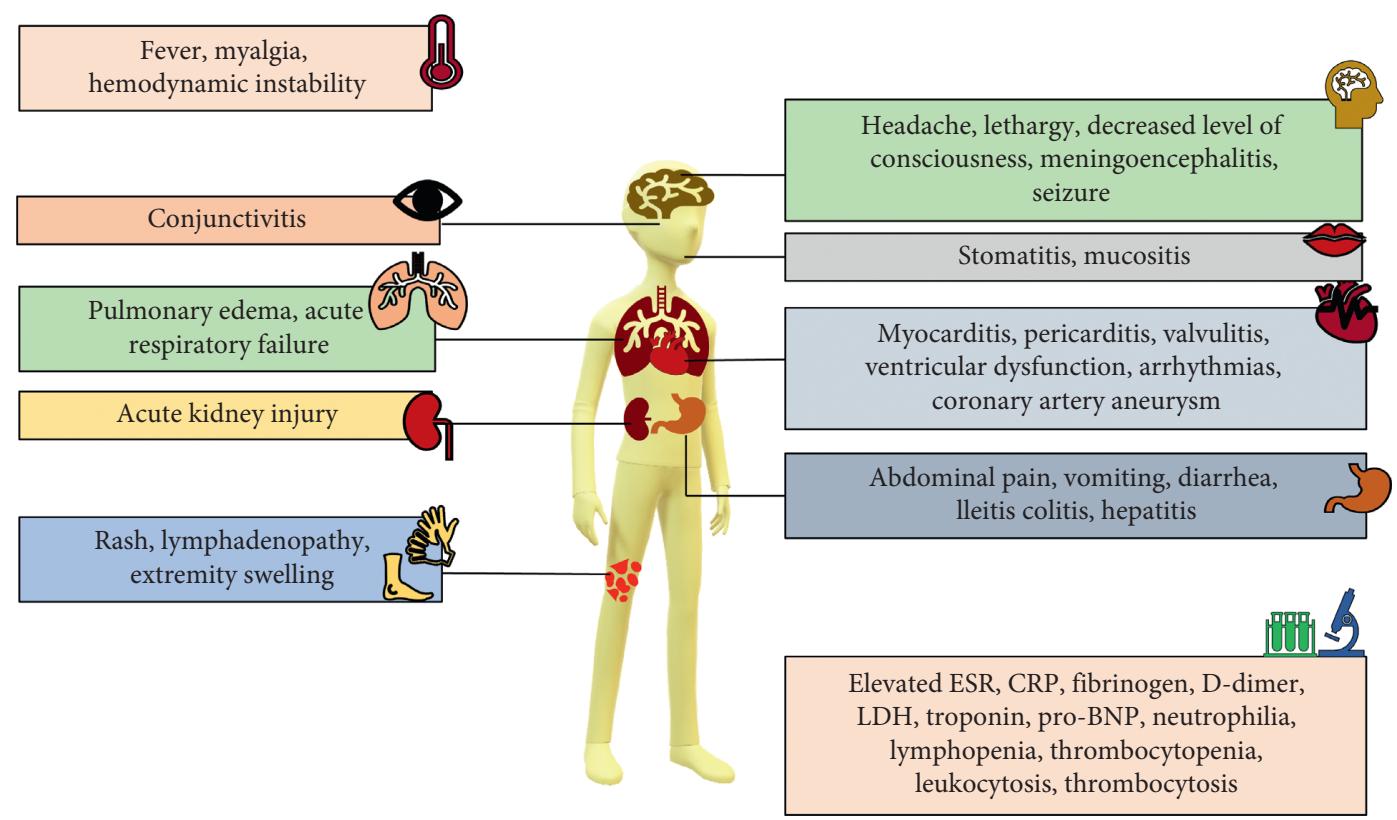

FIgURE 2: Clinical and laboratory manifestations of MIS-C. The most common and prominent features in patients with MIS-C include persistent fever, mucocutaneous manifestations, gastrointestinal symptoms, organ dysfunction, and significantly elevated inflammatory markers. The multiorgan effect of the SARS-CoV-2 is summarized in this figure.

Kawasaki disease alone with the median age being 9 years in the former. The presentation tends to begin around 4-6 weeks after contracting the virus, and they are usually PCR-negative. They commonly report fever and a spectrum of respiratory symptoms ranging from cough to dyspnea. $70 \%$ of patients with MIS-C also report gastrointestinal symptoms such as abdominal pain and diarrhea. Other signs and symptoms that are related to the Kawasaki-like features are rash, fissured lips, and conjunctivitis [18]. The cardiac abnormalities common in MIS-C are arrhythmia, conduction abnormalities, ventricular dysfunction, coronary artery dilatation or aneurysm, and pericarditis. According to multiple reports, the most common cardiac abnormality is LV dysfunction manifested by a depressed ejection fraction. Figure 2 contains a summary of the multiorgan involvement of SARS-CoV-2 in MIS-C.

While most patients are managed with inotropic support, a large cohort study revealed that around a third of the pediatric patients presenting with MIS-C required extracorporeal membrane oxygenation [64]. In a large case series, the majority of patients required inotropes but all of them recovered their LV function within a median of 2 days [72]. In another large pediatric-exclusive study including patients who developed MIS-C, the authors reported that $80 \%$ of them had cardiac involvement. The majority had elevated cardiac biomarkers (73\%), and $8 \%$ developed coronary artery aneurysms [26].

Patients with MIS-C may develop coronary artery abnormalities such as dilatations and aneurysms. Reports have demonstrated an array of descriptions ranging from small aneurysms to giant ones. While it has been hypothesized that a mechanism similar to that observed in Kawasaki disease occurs with MIS-C, the true pathophysiology behind coronary artery abnormalities has not been elucidated yet [78].
In addition to coronary artery abnormalities, the second most common cardiac abnormality in MIS-C is arrhythmia. First-degree heart block is the most common presentation of arrhythmia. It has been commonly reported in children presenting with LV dysfunction. Electrocardiogram reveals QT prolongation, ST-segment changes, or T-wave abnormalities [79]. Laboratory workup reveals elevated troponin, Pro-Brain Natriuretic Peptide (Pro-BPN), D-dimer, and inflammatory markers such as ferritin, erythrocyte sedimentation rate (ESR), interleukin-6 (IL-6), procalcitonin, and C-reactive protein (CRP). A complete blood count reveals neutrophilia, lymphopenia, and thrombocytopenia [64]. Chest X-ray displays cardiomegaly with possible hilar and mediastinal abnormalities. More than 50\% of these patients will have a degree of left ventricular dysfunction or failure noted on echocardiography. Global or wall hypokinesis has been documented as well. While right ventricular function is preserved in all patients, pericardial effusion is present in the minority of patients [22-31]. Only 15\% of patients with MIS-C will have CAA noted at the time of presentation. T1- and T2-weighted cardiac magnetic resonance imaging reveals diffuse increased intensity consistent with myocardial edema [18]. The natural course of MIS-C is not yet known. Ventricular dysfunction tends to improve over time. More studies are needed to elucidate the longterm manifestations of MIS-C.

5.8. Cardiac Involvement in Heart Transplant Patients. Cardiac transplant patients are a vulnerable population whose immunosuppressed state poses a risk of severe forms of COVID-19 infection. Despite their scarcity, reported cases and series on transplant recipients with COVID-19 show a heterogeneous clinical spectrum ranging from mild 
presentations to more severe forms [80-82]. The therapeutic rationale lies in maintaining a chronic immunosuppressed state while dampening the viral infection. Decreasing the dose of immunosuppressant drugs upon diagnosis is still debatable [81]. In one single-center series by Caraffa et al., 5 adult cardiac transplant patients hospitalized with SARSCoV-2 received a 50\% reduction in their immunosuppressant drug dose in addition to a medium dose of corticosteroids and antibiotics. One possible hypothesis is that the complete suspension of immunosuppressive drugs accompanied by viral-induced hyperinflammatory response may reactivate the immune memory causing potential graft rejection [80]. In another case series of 4 heart transplant patients, symptoms were mild and like the general population despite their immunosuppressed conditions. No adjustments of immunomodulatory drugs were made in this age group [81]. In a cohort study of 47 heart transplant recipients affected by COVID-19 in Northern Italy, $80 \%$ of the patients required hospitalization with adjustment of their immunosuppressive medication dose. Both the infection prevalence and case-fatality rate $(29 \%)$ were 2 -fold higher compared to the general population. No myocarditis was observed in any of the patients [80]. Latif et al. reported a similar case-fatality rate (25\%) in their cohort [83]. A survey in Wuhan, China, including 87 heart transplant patients reported a similar risk of SARS-CoV-2 infection as the general population provided preventive measures are properly practiced [84].

\section{Conclusion}

Cardiovascular involvement incurs significant prognostic value to patients diagnosed with COVID-19. Multidisciplinary teams with cardiology services are essential for managing all complications in patients suffering from COVID-19, as well as in patients with underlying cardiac diseases who are prone to severe and critical COVID-19. Ultimately, we argue that allocating sufficient resources for timely diagnosis and management of cardiac complications is crucial in reducing morbidity and mortality secondary to cardiovascular sequelae. In addition, performing a thorough evaluation with an electrocardiogram, echocardiography, and basic cardiac workup is essential for timely diagnosis and treatment.
Abbreviations
CAA: Coronary artery aneurysm
CRP: C-reactive protein
D: Days
ECMO: Extracorporeal membrane oxygenation
EF: $\quad$ Ejection fraction
ESR: $\quad$ Erythrocyte sedimentation rate
F: $\quad$ Females
IL1Ra: Interleukin-1 Receptor antagonist
IL-6: Interleukin-6
IL8: Interleukin-8
INR: International normalized ratio
IVIG: Intravenous immunoglobulin

LDH: Lactate dehydrogenase

LV: $\quad$ Left ventricle

M: $\quad$ Males

MR: $\quad$ Mitral regurgitation

NA: $\quad$ Not applicable

Pro-BNP: B-type natriuretic peptide

RV: $\quad$ Right ventricle

TNF alfa: Tumor necrosis factor alfa

USA: The United States of America.

\section{Data Availability}

Data are available upon request.

\section{Conflicts of Interest}

The authors have no conflicts of interest to declare.

\section{Authors' Contributions}

Tania Abi Nassif and Ghina Fakhri contributed equally to this manuscript.

\section{References}

[1] S. Perlman, "Another decade, another coronavirus," New England Journal of Medicine, vol. 382, no. 8, pp. 760-762, 2020.

[2] A. M. Zaki, S. van Boheemen, T. M. Bestebroer, A. D. M. E. Osterhaus, and R. A. M. Fouchier, "Isolation of a novel coronavirus from a man with pneumonia in Saudi Arabia," New England Journal of Medicine, vol. 367, no. 19, pp. 1814-1820, 2012.

[3] WHO, World Health Organization Coronavirus Disease 2019 (COVID-19): Situation Report-113, WHO, Geneva, Switzerland, 2020.

[4] N. K. Younis, R. O. Zareef, S. N. Al Hassan, F. Bitar, A. H. Eid, and M. Arabi, "Hydroxychloroquine in COVID-19 patients: pros and cons," Frontiers in Pharmacology, vol. 11, Article ID 597985, 2020.

[5] N. van Doremalen, T. Bushmaker, D. H. Morris et al., "Aerosol and surface stability of SARS-CoV-2 as compared with SARS-CoV-1," New England Journal of Medicine, vol. 382, no. 16, pp. 1564-1567, 2020.

[6] W. Tan and J. Aboulhosn, "The cardiovascular burden of coronavirus disease 2019 (COVID-19) with a focus on congenital heart disease," International Journal of Cardiology, vol. 309, pp. 70-77, 2020.

[7] C. Huang, Y. Wang, X. Li et al., "Clinical features of patients infected with 2019 novel coronavirus in Wuhan, China," The Lancet, vol. 395, no. 10223, pp. 497-506, 2020.

[8] B. Williams, "ESC/ESH guidelines for the management of arterial hypertension," Journal of Hypertension, vol. 39, no. 33, pp. 3021-3104, 2018.

[9] A. J. Turner, J. A. Hiscox, and N. M. Hooper, "ACE2: from vasopeptidase to SARS virus receptor," Trends in Pharmacological Sciences, vol. 25, no. 6, pp. 291-294, 2004.

[10] M. Gheblawi, K. Wang, A. Viveiros et al., "Angiotensinconverting enzyme 2: SARS-CoV-2 receptor and regulator of the renin-angiotensin system," Circulation Research, vol. 126, no. 10, pp. 1456-1474, 2020. 
[11] P. Zhou, X.-L. Yang, X.-G. Wang et al., “A pneumonia outbreak associated with a new coronavirus of probable bat origin," Nature, vol. 579, no. 7798, pp. 270-273, 2020.

[12] Z. Wu and J. M. McGoogan, "Characteristics of and important lessons from the coronavirus disease 2019 (COVID19) outbreak in China," JAMA, vol. 323, no. 13, pp. 1239-1242, 2020.

[13] R. Channappanavar and S. Perlman, "Pathogenic human coronavirus infections: causes and consequences of cytokine storm and immunopathology," Seminars in Immunopathology, vol. 39, no. 5, pp. 529-539, 2017.

[14] F. Zhou, T. Yu, R. Du et al., "Clinical course and risk factors for mortality of adult inpatients with COVID-19 in Wuhan, China: a retrospective cohort study," The Lancet, vol. 395, no. 10229, pp. 1054-1062, 2020.

[15] V. B. Patel, N. Clarke, Z. Wang et al., “Angiotensin II induced proteolytic cleavage of myocardial ACE2 is mediated by TACE/ADAM-17: a positive feedback mechanism in the RAS," Journal of Molecular and Cellular Cardiology, vol. 66, pp. 167-176, 2014.

[16] J. Boisrame-Helms, H. Kremer, V. Schini-Kerth, and F. Meziani, "Endothelial dysfunction in sepsis," Current Vascular Pharmacology, vol. 11, no. 2, pp. 150-160, 2013.

[17] X. M. Yuanyuan Dong, Y. Hu, X. Qi, F. Jiang, Z. Jiang, and S. Tong, "Epidemiology of COVID-19 among children in China," Pediatrics, vol. 145, no. 6, 2020.

[18] Y.-H. Loke, C. I. Berul, and A. S. Harahsheh, "Multisystem inflammatory syndrome in children: is there a linkage to Kawasaki disease?" Trends in Cardiovascular Medicine, vol. 30, no. 7, pp. 389-396, 2020.

[19] Y. Cui, M. Tian, D. Huang et al., "A 55-day-old female infant infected with 2019 novel coronavirus disease: presenting with pneumonia, liver injury, and heart damage," The Journal of Infectious Diseases, vol. 221, no. 11, pp. 1775-1781, 2020.

[20] D. Sun, "Clinical features of severe pediatric patients with coronavirus disease 2019 in Wuhan: a single center's observational study," World Journal of Pediatrics, vol. 16, no. 3, pp. 251-259, 2020.

[21] L. Su, X. Ma, H. Yu et al., "The different clinical characteristics of corona virus disease cases between children and their families in China-the character of children with COVID-19," Emerging Microbes \& Infections, vol. 9, no. 1, pp. 707-713, 2020.

[22] K. Joshi, D. Kaplan, A. Bakar et al., "Cardiac dysfunction and shock in pediatric patients with COVID-19," JACC: Case Reports, vol. 2, no. 9, pp. 1267-1270, 2020.

[23] J. Toubiana, C. Poirault, A. Corsia et al., "Kawasaki-like multisystem inflammatory syndrome in children during the covid-19 pandemic in Paris, France: prospective observational study," BMJ, vol. 369, p. m2094, 2020.

[24] M. P. Deza Leon, A. Redzepi, E. McGrath et al., "COVID-19associated pediatric multisystem inflammatory syndrome," Journal of the Pediatric Infectious Diseases Society, vol. 9, no. 3, pp. 407-408, 2020.

[25] E. M. Dufort, E. H. Koumans, E. J. Chow et al., "Multisystem inflammatory syndrome in children in New York state," New England Journal of Medicine, vol. 383, no. 4, pp. 347-358, 2020.

[26] L. R. Feldstein, E. B. Rose, S. M. Horwitz et al., "Multisystem inflammatory syndrome in U.S. children and adolescents," The New England Journal of Medicine, vol. 383, no. 4, pp. 334-346, 2020.

[27] M. Riollano-Cruz, "Multisystem inflammatory syndrome in children related to COVID-19: a New York City experience," Journal of Medical Virology, vol. 93, no. 1, pp. 424-433, 2020.
[28] Z. Belhadjer, M. Méot, F. Bajolle et al., "Acute heart failure in multisystem inflammatory syndrome in children in the context of global SARS-CoV-2 pandemic," Circulation, vol. 142, no. 5, pp. 429-436, 2020.

[29] T. Rogo, K. Mathur, and M. Purswani, "Systemic inflammation with cardiac involvement in pediatric patients with evidence of COVID-19 in a community hospital in the Bronx, New York," Journal of the Pediatric Infectious Diseases Society, vol. 9, no. 4, pp. 502-503, 2020.

[30] V. G. Jones, M. Mills, D. Suarez et al., "COVID-19 and Kawasaki disease: novel virus and novel case," Hospital Pediatrics, vol. 10, no. 6, pp. 537-540, 2020.

[31] A. Belot, "SARS-CoV-2-related paediatric inflammatory multisystem syndrome, an epidemiological study, France, 1 March to 17 May 2020," Eurosurveillance, vol. 25, no. 22, 2020.

[32] G. Giustino, S. P. Pinney, A. Lala et al., "Coronavirus and cardiovascular disease, myocardial injury, and arrhythmia," Journal of the American College of Cardiology, vol. 76, no. 17, pp. 2011-2023, 2020.

[33] R. O. Zareef, N. K. Younis, F. Bitar, A. H. Eid, and M. Arabi, "COVID-19 in pediatric patients: a focus on CHD patients," Frontiers in Cardiovascular Medicine, vol. 7, Article ID 612460, 2020.

[34] M. Böhm, N. Frey, E. Giannitsis, K. Sliwa, and A. M. Zeiher, "Coronavirus disease 2019 (COVID-19) and its implications for cardiovascular care: expert document from the German cardiac society and the World Heart Federation," Clinical Research in Cardiology, vol. 109, no. 12, pp. 1446-1459, 2020.

[35] W. Ni, X. Yang, J. Liu et al., "Acute myocardial injury at hospital admission is associated with all-cause mortality in COVID-19," Journal of the American College of Cardiology, vol. 76, no. 1, pp. 124-125, 2020.

[36] F. Jiang, J. Yang, Y. Zhang et al., "Angiotensin-converting enzyme 2 and angiotensin 1-7: novel therapeutic targets," Nature Reviews Cardiology, vol. 11, no. 7, pp. 413-426, 2014.

[37] H. Jia, "Pulmonary angiotensin-converting enzyme 2 (ACE2) and inflammatory lung disease," Shock, vol. 46, no. 3, pp. 239-248, 2016.

[38] V. Giacomet, V. A. Manfredini, G. Meraviglia et al., “Acute inflammation and elevated cardiac markers in a two-month-old infant with severe acute respiratory syndrome coronavirus 2 infection presenting with cardiac symptoms," Pediatric Infectious Disease Journal, vol. 39, no. 7, pp. e149-e151, 2020.

[39] S. Samuel, R. A. Friedman, C. Sharma et al., "Incidence of arrhythmias and electrocardiographic abnormalities in symptomatic pediatric patients with PCR-positive SARSCoV-2 infection, including drug-induced changes in the corrected QT interval," Heart Rhythm, vol. 17, no. 11, pp. 1960-1966, 2020.

[40] M.-L. Oberweis, A. Codreanu, W. Boehm et al., "Pediatric lifethreatening coronavirus disease 2019 with myocarditis," Pediatric Infectious Disease Journal, vol. 39, no. 7, pp. e147-e149, 2020.

[41] W. Xia, J. Shao, Y. Guo, X. Peng, Z. Li, and D. Hu, "Clinical and CT features in pediatric patients with COVID-19 infection: different points from adults," Pediatric Pulmonology, vol. 55, no. 5, pp. 1169-1174, 2020.

[42] T. Guo, Y. Fan, M. Chen et al., "Cardiovascular implications of fatal outcomes of patients with coronavirus disease 2019 (COVID-19)," JAMA Cardiology, vol. 5, no. 7, pp. 811-818, 2020.

[43] S. Shi, M. Qin, B. Shen et al., "Association of cardiac injury with mortality in hospitalized patients with COVID-19 in 
Wuhan, China," JAMA Cardiology, vol. 5, no. 7, pp. 802-810, 2020.

[44] Q. Ruan, K. Yang, W. Wang, L. Jiang, and J. Song, "Clinical predictors of mortality due to COVID-19 based on an analysis of data of 150 patients from Wuhan, China," Intensive Care Medicine, vol. 46, no. 5, pp. 846-848, 2020.

[45] B. Li, J. Yang, F. Zhao et al., "Prevalence and impact of cardiovascular metabolic diseases on COVID-19 in China," Clinical Research in Cardiology, vol. 109, no. 5, pp. 531-538, 2020.

[46] G. Y. Oudit, Z. Kassiri, C. Jiang et al., "SARS-coronavirus modulation of myocardial ACE2 expression and inflammation in patients with SARS," European Journal of Clinical Investigation, vol. 39, no. 7, pp. 618-625, 2009.

[47] M. R. Mehra, S. S. Desai, S. Kuy, T. D. Henry, and A. N. Patel, "Cardiovascular disease, drug therapy, and mortality in covid19," New England Journal of Medicine, vol. 382, no. 25, p. e102, 2020

[48] I.-C. Kim, J. Y. Kim, H. A. Kim, and S. Han, “COVID-19-related myocarditis in a 21-year-old female patient," European Heart Journal, vol. 41, no. 19, p. 1859, 2020.

[49] R. M. Inciardi, L. Lupi, G. Zaccone et al., "Cardiac involvement in a patient with coronavirus disease 2019 (COVID19)," JAMA Cardiology, vol. 5, no. 7, pp. 819-824, 2020.

[50] G. Tavazzi, C. Pellegrini, M. Maurelli et al., "Myocardial localization of coronavirus in COVID-19 cardiogenic shock," European Journal of Heart Failure, vol. 22, no. 5, pp. 911-915, 2020.

[51] P. Wenzel, S. Kopp, S. Göbel et al., "Evidence of SARS-CoV-2 mRNA in endomyocardial biopsies of patients with clinically suspected myocarditis tested negative for COVID-19 in nasopharyngeal swab," Cardiovascular Research, vol. 116, no. 10, pp. 1661-1663, 2020.

[52] D. Lindner, A. Fitzek, H. Bräuninger et al., "Association of cardiac infection with SARS-CoV-2 in confirmed COVID-19 autopsy cases," JAMA Cardiology, vol. 5, no. 11, pp. 1281-1285, 2020.

[53] D. Wang, B. Hu, C. Hu et al., "Clinical characteristics of 138 hospitalized patients with 2019 novel coronavirus-infected pneumonia in Wuhan, China," JAMA, vol. 323, no. 11, pp. 1061-1069, 2020.

[54] A. Lala, K. W. Johnson, J. L. Januzzi et al., "Prevalence and impact of myocardial injury in patients hospitalized with COVID-19 infection," Journal of the American College of Cardiology, vol. 76, no. 5, pp. 533-546, 2020.

[55] C. Bavishi, R. O. Bonow, V. Trivedi, J. D. Abbott, F. H. Messerli, and D. L. Bhatt, "Special article-acute myocardial injury in patients hospitalized with COVID-19 infection: a review," Progress in Cardiovascular Diseases, vol. 63, no. 5, pp. 682-689, 2020.

[56] K. Thygesen, J. S. Alpert, A. S. Jaffe et al., "Fourth universal definition of myocardial infarction (2018)," Journal of the American College of Cardiology, vol. 72, no. 18, pp. 2231-2264, 2018.

[57] P. Libby, J. Loscalzo, P. M. Ridker et al., "Inflammation, immunity, and infection in atherothrombosis," Journal of the American College of Cardiology, vol. 72, no. 17, pp. 2071-2081, 2018.

[58] W. Lim, "Elevated cardiac troponin measurements in critically ill patients," Archives of Internal Medicine, vol. 166, no. 22, pp. 2446-2454, 2006.

[59] L. Sarkisian, L. Saaby, T. S. Poulsen et al., "Prognostic impact of myocardial injury related to various cardiac and noncardiac conditions," The American Journal of Medicine, vol. 129, no. 5, pp. 506-514, 2016.

[60] L. Sarkisian, L. Saaby, T. S. Poulsen et al., "Clinical characteristics and outcomes of patients with myocardial infarction, myocardial injury, and nonelevated troponins," The American Journal of Medicine, vol. 129, no. 4, pp. 446-e21, 2016.

[61] T. Chen, D. Wu, H. Chen et al., "Clinical characteristics of 113 deceased patients with coronavirus disease 2019: retrospective study," BMJ, vol. 368, p. m1091, 2020.

[62] D. R. Lakkireddy, "Guidance for cardiac electrophysiology during the COVID-19 pandemic from the heart Rhythm society COVID-19 task force; electrophysiology section of the American college of cardiology; and the electrocardiography and arrhythmias committee of the council on clinical cardiology, American heart association," Circulation, vol. 141, no. 21, pp. e823-e831, 2020.

[63] H. Xu, "Clinical characteristics and risk factors of cardiac involvement in COVID-19," Journal of the American Heart Association, vol. 9, no. 18, Article ID e016807, 2020.

[64] T. Alsaied, A. H. Tremoulet, J. C. Burns et al., "Review of cardiac involvement in multisystem inflammatory syndrome in children," Circulation, vol. 143, no. 1, pp. 78-88, 2021.

[65] K. Sud, B. Vogel, C. Bohra et al., "Echocardiographic findings in patients with COVID-19 with significant myocardial injury," Journal of the American Society of Echocardiography, vol. 33, no. 8, pp. 1054-1055, 2020.

[66] H. M. Mahmoud-Elsayed, W. E. Moody, W. M. Bradlow et al., "Echocardiographic findings in patients with COVID-19 pneumonia," Canadian Journal of Cardiology, vol. 36, no. 8, pp. 1203-1207, 2020.

[67] Y. Szekely, Y. Lichter, P. Taieb et al., "Spectrum of cardiac manifestations in COVID-19," Circulation, vol. 142, no. 4, pp. $342-353,2020$.

[68] J. A. Fried, K. Ramasubbu, R. Bhatt et al., "The variety of cardiovascular presentations of COVID-19," Circulation, vol. 141, no. 23, pp. 1930-1936, 2020.

[69] M. S. Besler and H. Arslan, "Acute myocarditis associated with COVID-19 infection," The American Journal of Emergency Medicine, vol. 38, no. 11, pp. 2489 e1-2489 e2, 2020.

[70] J. A. Luetkens, A. Isaak, S. Zimmer et al., "Diffuse myocardial inflammation in COVID-19 associated myocarditis detected by multiparametric cardiac magnetic resonance imaging," Circulation: Cardiovascular Imaging, vol. 13, no. 5, Article ID e010897, 2020.

[71] V. O. Puntmann, M. L. Carerj, I. Wieters et al., "Outcomes of cardiovascular magnetic resonance imaging in patients recently recovered from coronavirus disease 2019 (COVID19)," JAMA Cardiology, vol. 5, no. 11, pp. 1265-1273, 2020.

[72] M. Grimaud, J. Starck, M. Levy et al., "Acute myocarditis and multisystem inflammatory emerging disease following SARSCoV-2 infection in critically ill children," Annals of Intensive Care, vol. 10, no. 1, p. 69, 2020.

[73] M. Harada, Y. Yokouchi, T. Oharaseki et al., "Histopathological characteristics of myocarditis in acute-phase Kawasaki disease," Histopathology, vol. 61, no. 6, pp. 1156-1167, 2012.

[74] C. Qin, L. Zhou, Z. Hu et al., "Dysregulation of immune response in patients with coronavirus 2019 (COVID-19) in Wuhan, China," Clinical Infectious Diseases, vol. 71, no. 15, pp. 762-768, 2020.

[75] N. Pathan, C. A. Hemingway, A. A. Alizadeh et al., "Role of interleukin 6 in myocardial dysfunction of meningococcal septic shock," The Lancet, vol. 363, no. 9404, pp. 203-209, 2004. 
[76] I. A. Hobai, J. Edgecomb, K. LaBarge, and W. S. Colucci, "Dysregulation of intracellular calcium transporters in animal models of sepsis-induced cardiomyopathy," Shock, vol. 43, no. 1, pp. 3-15, 2015.

[77] WHO, Multisystem Inflammatory Syndrome in Children and Adolescents Temporally Related to COVID-19, WHO, Geneva, Switzerland, 2020.

[78] I. Valverde, Y. Singh, J. Sanchez-de-Toledo et al., "Acute cardiovascular manifestations in 286 children with multisystem inflammatory syndrome associated with COVID-19 infection in Europe," Circulation, vol. 143, no. 1, pp. 21-32, 2021.

[79] E. Blondiaux, P. Parisot, A. Redheuil et al., "Cardiac MRI in children with multisystem inflammatory syndrome associated with COVID-19," Radiology, vol. 297, no. 3, pp. E283-E288, 2020.

[80] T. Bottio, L. Bagozzi, A. Fiocco et al., "COVID-19 in heart transplant recipients," JACC: Heart Failure, vol. 9, no. 1, pp. 52-61, 2021.

[81] H. Lee, B. S. Mantell, M. E. Richmond et al., "Varying presentations of COVID-19 in young heart transplant recipients: a case series," Pediatric Transplantation, vol. 24, no. 8, Article ID e13780, 2020.

[82] F. Li, J. Cai, and N. Dong, "First cases of COVID-19 in heart transplantation from China," The Journal of Heart and Lung Transplantation, vol. 39, no. 5, pp. 496-497, 2020.

[83] F. Latif, M. A. Farr, K. J. Clerkin et al., "Characteristics and outcomes of recipients of heart transplant with coronavirus disease 2019," JAMA Cardiology, vol. 5, no. 10, pp. 1165-1169, 2020.

[84] Z.-L. Ren, R. Hu, Z.-W. Wang et al., "Epidemiologic and clinical characteristics of heart transplant recipients during the 2019 coronavirus outbreak in Wuhan, China: a descriptive survey report," The Journal of Heart and Lung Transplantation, vol. 39, no. 5, pp. 412-417, 2020. 